292

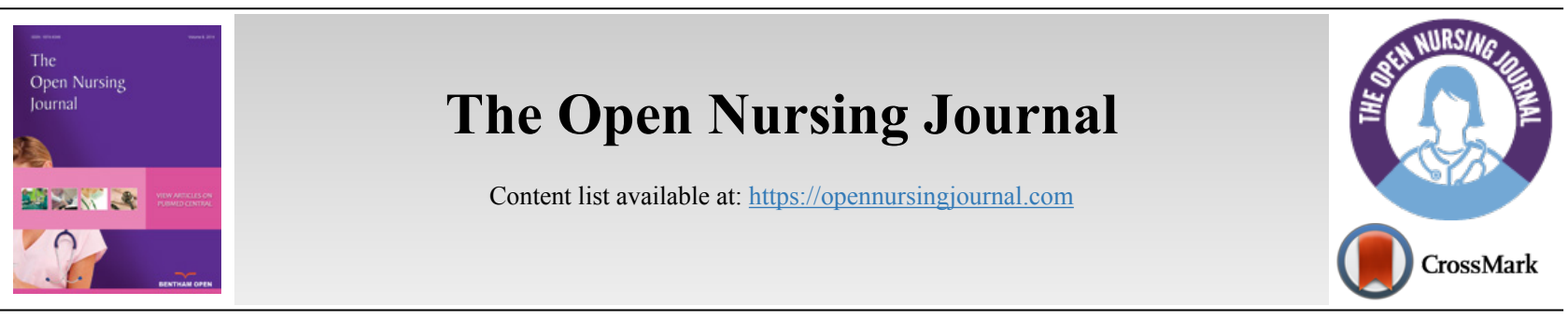

RESEARCH ARTICLE

\title{
Challenges Midwives in Limpopo Province Encounter when Implementing Saving Mothers Recommendations
}

\author{
Kobel Elizabeth Mothapo ${ }^{1}$, Maria Sonto Maputle ${ }^{1, *}$, Hilda N Shilubane ${ }^{1}$ and Lizzy Netshikweta ${ }^{1}$ \\ ${ }^{1}$ Department of Advanced Nursing, University of Venda, Private Bag X5050, Thohoyandou 0950, South Africa
}

\begin{abstract}
:
Introduction:

The recommendations of Saving Mothers report by the National Committee for Confidential Enquiry into Maternal Deaths (NCCEMD) were formulated in response to the high maternal mortality rate in South Africa. Saving Mothers' Report recommendations were implemented in maternity units of Limpopo province. However, the mortality rate for 2014-2016 in Limpopo was 165.16/100000 live births against the national target of 38/100000 live births. The purpose of this study was to explore the challenges faced by registered midwives when implementing these recommendations.

Methods:

A qualitative research approach, which is descriptive, exploratory and contextual design, was used. A non-probability, purposive and convenience sampling was used to select 25 participants. Data were collected from August to October 2017 through the individual in-depth unstructured interview from 22 participants based on data saturation. Trustworthiness was ensured through credibility, dependability, transferability and confirmability. Data were analysed using Tesch's open coding technique. Ethical standards were adhered to.
\end{abstract}

Results:

The findings revealed one theme and five sub-themes indicating hindrances to the implementation of recommendations of Saving Mothers Report by midwives in maternity units in Limpopo province.

Conclusion:

The researchers concluded that the registered midwives were faced with challenges that need to be addressed to facilitate the implementation of recommendations for Saving Mothers report effectively. Further research was recommended with implications in practice and nursing education.

Keywords: Challenges, Implementation, Recommendations, Saving mothers, Maternal Deaths (NCCEMD), MMR.

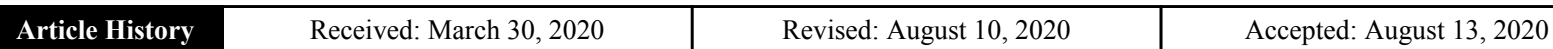

\section{INTRODUCTION}

The National Committee for Confidential Enquiries into Maternal Deaths (NCCEMD) in South Africa (SA), compiled first "Saving Mothers Report" in 1998. The report had recommendations that intended to reduce maternal mortality rate (MMR) [1]. Due to implementing of recommendations, the number of maternal deaths gradually reduced at each triennium; from $12.8 \%$ in $2008-2010$ to $2011-2013$ and $12.5 \%$ from 2011-2013 to 2014-2016. The report revealed the pattern of disease-causing maternal deaths, the avoidable factors, mis-

\footnotetext{
* Address correspondence to this author at Department of Advanced Nursing, University of Venda, Private Bag X5050, Thohoyandou 0950, South Africa; Tel: +27159628125; E-mail: sonto.maputle@univen.ac.za
}

sed opportunities and substandard care related to these deaths in South Africa as a country and its provinces [2].

The five leading causes of maternal deaths in Limpopo Province in the Triennium 2008-2010 were non-pregnancy related infections which amounted to $(35.6 \%)$, obstetric haemorrhage amounting (17.6\%), hypertensive disorders (13.8\%), pre-existing medical and surgical disorders (10.2\%) and anaesthetic complications (5.7\%). The statistics for MMR indicates that Limpopo Province is far above the national target for 2015 of 38/100 000. In 2014-2016, Limpopo Province had the MMR of 165.16 per 100000 live births irrespective of implementing the recommendations of Saving Mothers' Report. The results by the National Department of Health indicate that in 2017, MMR in Limpopo was 181 per 100000 
live births. The MMR was found to be escalating, whereas the recommendations were implemented.

Haemorrhage has been found to be the major reason for maternal deaths [3 - 5]. It was pointed in the National Department of Health that considerable efforts have been put into decreasing deaths from obstetric haemorrhage which was demonstrated by a decline of $2 \%$ in 2017 . However, deaths owing to complications of hypertension remained high [1]. An assessment of maternal and perinatal deaths owing to complications of hypertension involves two major factors, namely, failure to refer women with hypertension, mostly from the primary healthcare clinics, and infrequent antenatal visits to detect sudden changes in a woman's condition. The registered midwives are major implementers of the recommendations as they are providers of care during pregnancy, labour and postnatal period. It was not clear whether midwives adhered to implementing the protocol and clear referral criteria for hypertensive disorders. There is still much to do, and efforts need to concentrate on improving the health system to reduce the deaths owing to hypertension.

The Hs recommendations were the focus in the current study, namely, HIV and TB, obstetric Haemorrhage, Hypertensive disorders, Health worker training and Health system strengthening [5]. The midwives are expected to continue implementing the HIV care protocols since it is documented that two-thirds of maternal deaths were to HIV positive women. The success of the antiretroviral treatment programme for HIV-positive women contributed to a decline in maternal deaths [1]. TB has been found to be the most cause of death in non-pregnancy related infections [4]. Hence, all pregnant women irrespective of positive HIV status should be screened for TB. It is further emphasised that pregnant HIV positive women should be screened for TB at each interaction during pregnancy or the postnatal period. The prophylaxis of Isoniazid is prescribed or to fast-track treatment if TB is diagnosed.

Limpopo Province is a rural area where most of health facilities are resourced limited. Poor implementation of Emergency Obstetric Care (EOC), policies, fragmented care, limited referral systems, non-availability of obstetricians, and inappropriate delegation of lifesaving functions to medical officers and nurses were the cause of concern. Apart from the MMR statistics provided above, registered midwives highlighted the challenges they encounter when implementing the recommendations. This was confirmed by the findings of a study conducted in Australia by Bar-Zeev [6,7], which found that resourcing organisation of health services, the beliefs, attitudes and practices of midwives were the major factors affecting the provision of quality care. The best practices were learned from the results of a study done in the United States of America, which revealed that they managed to reduce MMR and improve maternal health outcomes by implementing the national management protocol. Reducing MMR crucially depends upon ensuring that women have access to quality care and midwives are implementing the Saving Mothers report recommendations during childbirth, during labour and postnatally [8]. All health workers involved in maternity care must be trained in the Essential Steps in Managing Obstetric
Emergencies - Emergency Obstetric Simulation Training (ESMOE-EOST) programme, obstetric anaesthetic module, HIV counselling, testing and support, initiation and monitoring of HAART, TB care and the recognition, assessment, diagnosis and treatment of severe respiratory infections. This will empower them to implement the recommendations. The health system should be strengthened; there should be 24-hour access to functioning emergency obstetric care (both basic and comprehensive). However, like in other provinces in South Africa, Limpopo was experiencing a limited number of skilled staff at district hospitals. The purpose of this study was to explore challenges faced by registered midwives when implementing recommendations of the Saving Mothers' Report in the maternity units of Limpopo Province.

\section{METHODS}

The study was conducted at selected hospitals and clinics in Limpopo Province (Fig. 1).

The study was conducted in 2017. A qualitative, exploratory-descriptive contextual design was used. The design was deemed appropriate to investigate the challenges experienced by registered midwives in relation to implementing the recommendations of the Saving Mothers Report. Non-probability, purposive sampling was used to select five (5) hospitals, one hospital in each health district. Hospitals with the high MMR for that period were selected, namely; Tshilidzini, Matlala, Pietersburg, Voortrekker Memorial and Kgapane. Convenience sampling was used to sample twentyfive (25) registered midwives. Registered midwives with two or more years of experience were included as participants. The researcher visited the unit as per appointment for obtaining the consent from the participants. Data were collected from August to October 2017 through one-on-one in-depth interviews. The individual interview, which lasted 30-45 minutes, was conducted in a quiet room using the English language. A central question asked was "What challenges do you experience when implementing the recommendations of the Saving Mothers Report in this facility". A voice recorder was used to capture the conversation. Probing was done to get more information from participants. Data were collected until saturation, where no new information emerged at number twenty-two (22) (Table 1). Data were analysed manually through Tesch's eight steps. An inductive, descriptive opencoding technique was used [9]. The steps followed were, getting a sense of the whole, picking out one most interesting transcribed interview document, making a list of topics, clustering together similar topics, abbreviating the topics as codes, looking for the most descriptive wording, assembling data belonging together that came up with one theme and five sub-themes described in Table 2. Trustworthiness was ensured through the four principles of Lincoln and Guba's framework, as described in the study [10 - 12]. Credibility was ensured by a prolonged engagement, which increased rapport and to clarify descriptions with participants through familiarity. Data triangulation was ensured by using different data collection methods through field notes and in-depth individual interviews referential adequacy by the promoters and co-coder, an independent person, who was a midwife and experienced qualitative researcher. Confirmability was ensured by audit 
trail of voice recorder and the field notes to determine the conclusions, interpretations and recommendations if traced from their sources. Transferability was ensured through a thick description of the research methods and design.

Ethical standards were ensured by obtaining the ethical clearance (Ref: SHS/16/PDC/01/2804), from the University of Venda Ethics Committee, permission to conduct the study from the Limpopo Provincial Department of Health, the District Managers of the districts, the hospital CEOs and the participants. Participants gave written, informed consent, and were informed of their right to withdraw from the study without any penalty. Ethical principles of fairness, privacy, confidentiality, anonymity as well as participants' rights to voluntarily participate in the study were adhered to.

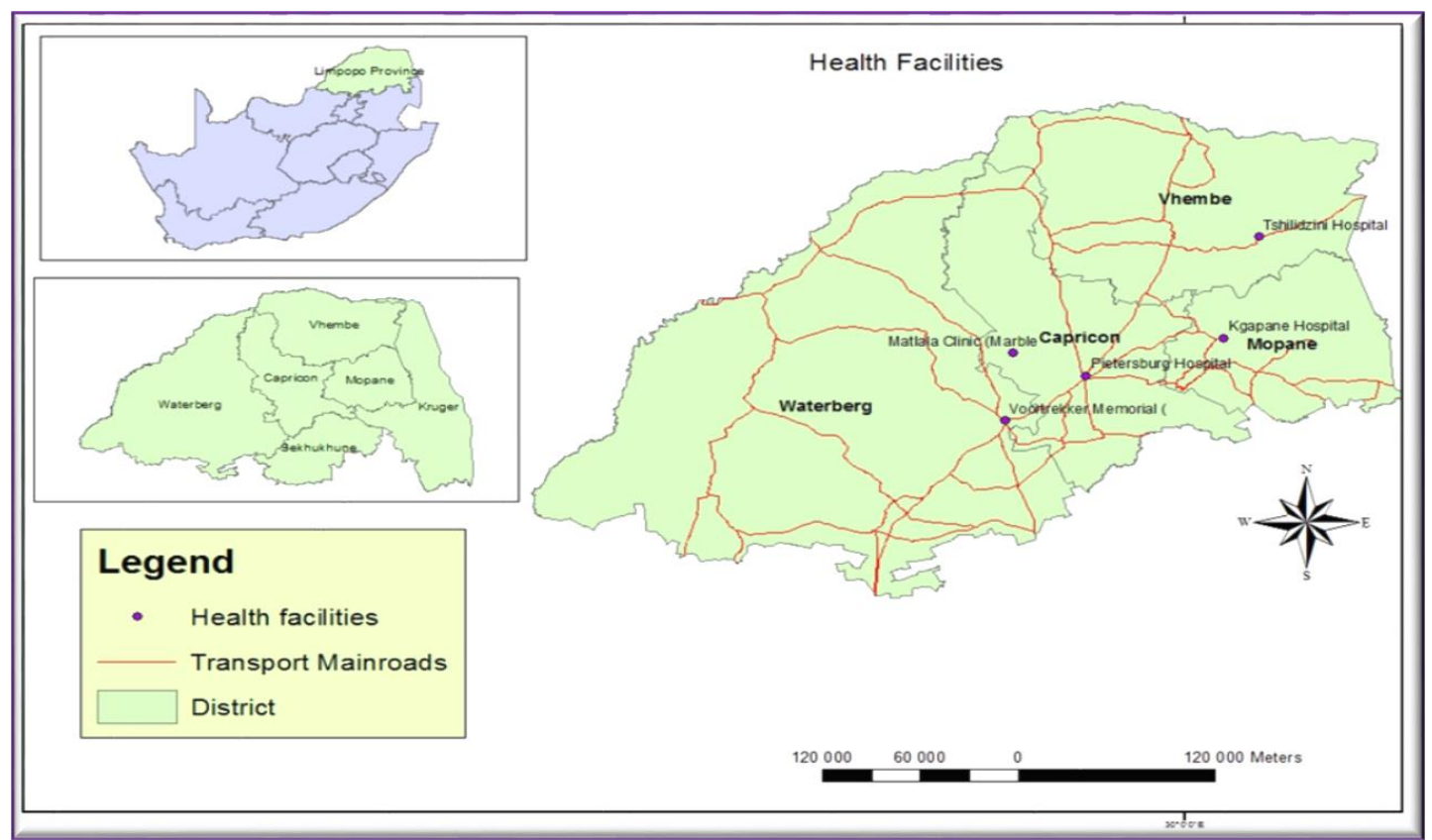

Fig. (1). Limpopo Province districts and health facilities.

Table 1. Demographic characteristics of participants $(n=22)$.

\begin{tabular}{|c|c|c|}
\hline Demographic & Number & Percentage (\%) \\
\hline \multicolumn{3}{|l|}{ Gender } \\
\hline Males & 2 & 9 \\
\hline Females & 20 & 91 \\
\hline \multicolumn{3}{|l|}{ Age of registered midwives } \\
\hline$>30$ years & 7 & 31.8 \\
\hline $30-39$ years & 3 & 13.6 \\
\hline $40-49$ years & 8 & 36.6 \\
\hline $50-60$ years & 4 & 18.1 \\
\hline \multicolumn{3}{|l|}{ Years of experience } \\
\hline $3-9$ & 12 & 55 \\
\hline $10-15$ & 6 & 27 \\
\hline 16 and above & 4 & 18 \\
\hline \multicolumn{3}{|l|}{ Position held } \\
\hline Registered nurse & 16 & 73 \\
\hline Operational manager & 2 & 9 \\
\hline District manager & 4 & 18 \\
\hline \multicolumn{3}{|l|}{ Qualifications } \\
\hline Diploma in Nursing & 10 & 45 \\
\hline Bachelor degree in Nursing & 4 & 18 \\
\hline Diploma in Advanced Midwifery & 8 & 36 \\
\hline
\end{tabular}


Table 2. Theme and sub-themes that emerged.

\begin{tabular}{|c|c|}
\hline Theme & Sub-themes \\
\hline \multirow{5}{*}{$\begin{array}{l}\text { 1. Hindrances to the implementation } \\
\text { of recommendations of Saving } \\
\text { Mothers Report in maternity units }\end{array}$} & 1.1. Poor supply of resources interfered with implementation of recommendations for Saving Mothers report \\
\hline & $\begin{array}{l}\text { 1.2. Increased workload that prevented the limited number of (available) midwives from providing quality } \\
\text { care to patients }\end{array}$ \\
\hline & 1.3. Failure to implement referral system protocol by health professionals \\
\hline & 1.4. Non-adherence to protocols of early antenatal booking by pregnant women \\
\hline & $\begin{array}{l}\text { 1.5. Perceived lack of appreciation by management when midwives took an extra mile in performing their } \\
\text { duties }\end{array}$ \\
\hline
\end{tabular}

\section{FINDINGS}

Twenty-two participants, of which two were males and twenty female, registered midwives, were interviewed, as indicated in Table 1 below. Females are dominating because historically, nursing has been predominately a female profession.

One main theme with five sub-themes emerged, indicating hindrances to the implementation of recommendations of Saving Mothers Report by midwives.

3.1. Theme: Hindrances to the Implementation of Recommendations of Saving Mothers Report in Maternity Units

Midwives were trained to provide care to pregnant women and to adhere to protocols, however, they cited hindrances faced, and this was supported by the following quote by a participant who said:

"Eish... The recommendations are ideal to improve maternal health, but we face problems when we are expected to implement them. We experience a lot of barriers, which are really frustrating." (30yrs, female, number 1)

The sub-themes that emerged from this theme are described below;

\subsection{Sub-theme 1.1: Poor Supply of Resources Interfered with the Implementation of Recommendations for Saving Mothers Report}

To implement recommendations, adequate resources are required; however, in the maternal health units, poor supply of resources was experienced as cited by a participant who said:

\footnotetext{
"There is a great shortage of blood. Blood for transfusion is scarce and it takes a long time to get access to it. You will be expected to refer a patient for blood transfusion, whereas to save life, this could be done quickly at the facility to save lives." (29 yrs. female, number 7)
}

\begin{abstract}
"Medications like Ferrous Sulphate, Folic Acid and Calcium supplements are not available at the clinics. Patients are encouraged to buy for themselves, which they may not and we will only notice when the woman is not improving on subsequent visit or when the woman presents with complications. The ratio of registered midwives to patients is 1: 30, hampering patients' observation. Equipment like scan machine, mobile X-Ray machine is also not available." (35 yrs. female, number 8)
\end{abstract}

\subsection{Sub-theme 1.2: Increased Workload that Prevented the Limited Number of (available) Midwives from Providing Quality Care to Patients}

The workload needs to be proportional to the number of staff on duty so that complications may be early detected and avoided. The results indicated that there was an overload of work as patients were referred while they could have managed at primary health care; patients were also self-referring to the unit. One participant cited the quote by saying:

\begin{abstract}
"We have high-risk patients in this very same over-crowded ward who needs to be monitored frequently and they end up complicating due to lack of frequent monitoring as midwives will be dealing with the normal deliveries who should be done at clinics. Staff at the clinics refer the patients with a diagnosis which do not correlate with what is found on arrival to the hospital. Patients refer themselves to the hospital, causing overcrowding."(38 yrs, female, number 16).
\end{abstract}

\subsection{Sub-theme 1.3: Failure to Implement Referral System Protocol by Health Professionals}

Recommendations of Saving Mothers' Report indicate that there should be clear referral routes and criteria for women to survive. However, there was still a challenge of referrals between health facilities. This was confirmed by a participant who said: 
have been transferred from other hospitals and the reason is that doctors who transfer do not check patients before transfer. Patients come with no blood results. The women from PHC also just come in (self-referral) saying the clinic is not working during the night" (44 yrs, male, number 3)

Another participant supported the non-adherence to referral protocol by saying:

"The referring doctors do not write all patients
information and doctors have to call back to
find out what was done and what was not done
to the patient. They send patients late. At other
times they send patients with no results, no
sonar done. Delays are caused as patients need
to be checked for things that could have been
done at the transferring hospital. The good
thing is that they start the Magnesium
Sulphate for patients with pre-eclampsia". ( 27
yrs. female, number 18)

\subsection{Sub-theme 1.4: Non-adherence to the Protocol of Early Antenatal Care Booking by Pregnant Women}

The Basic Antenatal Care protocol recommends that pregnant women should book for antenatal as soon as they miss the period. In this study, it emerged that women were booking during the second or third trimester. When booking late, it becomes difficult to implement the recommendations. This was confirmed by a participant when saying:

"The older women are shy to attend a clinic
with the young ones. They book late or not at
all giving reasons like" I was not aware that I
was pregnant" How do you then manage such
a patient because even if you want to adhere to
the protocols they booked late and made it
difficult to implement the Saving Mothers
Report and there is nobody to apply them on.
Some deliver at home and some still practice
non-acceptable practice, like pica or adhering
to cultural practices." Sighing! ( 42 yrs, male,
19)

3.6. Sub-theme 1.5: Perceived Lack of Appreciation by Management when Midwives took an Extra mile in Performing their Duties

The recommendation number 4 of the 5 Hs encourages the strengthening of the health systems. The results showed that the management did not appreciate and appraise midwives. This was noticed when a participant said:

"There is low morale of staff as complaints about shortage are not attended to. There are also no compliments from management for hard work. Staff members who work more are not rewarded by the performance management system. So, when one is burnout, just work routinely" (33 yrs, female, number 1).

\section{RESULTS AND DISCUSSION}

The findings revealed that participants perceived poor supply of both human and material resources, which affected the ability of registered midwives on adhering to protocols. The poor supply of resources affects the effective implementation of recommendations; thus, the MMR will not be reduced. Recommendation 6 in the National Guidelines [12] states, "Blood must be available at every institution where caesarean sections are performed. However, health facilities were experiencing a shortage of blood." The situation has not improved as Gallaher [13] also found that lack of availability of blood transfusion at facilities, and this resulted in $1.7 \%$ cases of maternal mortality occurring due to requirement for urgent blood transfusions which were not accessed. Citation by du Plessis [14] further found that the use of blood and blood products for obstetric haemorrhage is lifesaving, however, in the developing countries, there seemed to be increasing obstetric haemorrhage which is still one of the leading causes of maternal deaths. The current findings are in line with guidelines on managing drug product shortages (ASHP), which indicated that the drug shortage tripled from 2004 to 2014 with many shortages reported to be due to business decisions and disrupted supply as a result of quality control problems $[15$, 16]. Similarly, the results from the Commission of Enquiry into the Free State Health Care System in 2015 found that shortages and stock-outs of medication and medical supplies were chronic and were endangering the lives and health of vulnerable people across the province [17]. Clients were not given iron preparations since they were not available but advised to buy. It was reported that some clients could not afford and this predisposed them to develop anaemia. The unavailability of iron supplements discouraged clients from accessing and trusting the health care system. They further indicated that there were insufficient human resources, which prevent the fulfillment of the right of access to health care services. However, this was contradicting the findings of the study by Phala [18], where the facilities were found to have met the requirements as stipulated in the World Health Organisation [19]; the equipment needed at facilities were available. The shortage of human and material resources negatively affected the implementation of the recommendations in Limpopo province.

The findings of the study revealed that participants experienced increased workload due to an increased number of admissions, which resulted from self-referral as most PHC facilities were not conducting deliveries. Some facilities were staffed with qualified Advanced Midwives, however, overcrowding in these units lead to the provision of substandard care. The provision of quality health care is a key aspect of governmental policies [19]. World Health Organisation [19] further pointed out that a shortage of skilled birth attendants in developing countries remains a challenge to 
improve maternal health outcomes. Recommendations 4 and 5 could assist in saving the lives of mothers by training enough health care professionals as well as strengthening the health system by conducting frequent in-service training. The authors Chopra et al. [20] in Saving Mothers and Saving Babies campaign concurred that perinatal care indices of quality care that depend on the availability of adequate resources at the facilities and staff with the right skills mix and knowledge to manage clients.

The key Recommendation number 2 in the National Department of Health [3] states that referral routes and criteria for referral must be developed and implemented. However, this was still a challenge between health facilities. The Limpopo province referral protocol states that only consultants can discuss patients with referring institutions and that all patients should come with their blood results. The forms for referral should be completed and arrangements with the receiving facility should be made following the correct communication channels. But the current findings revealed that midwives would transfer patients with invalid reasons to the hospital; and those patients would deliver normally without complications. Therefore, registered midwives did not adhere to the referral system suggested in the recommendations for Saving Mothers Report, which could be fuelled by a lack of referral criteria. Failure to develop and adhere to the referral criteria could mean that mothers will continue to die in the hospital as those with problems might be missed and not monitored due to overcrowding. Authors [21] in the study conducted in Australia agreed with these findings when indicating that there were still deficiencies in referral procedures between the transferring and the referral hospital, which needed attention because agreement should be developed so that it could be followed during transfers/ referral. This was confirmed by Kiruja [22], who pointed reasons for late arrival to the referral hospital as poor knowledge, poor communication and problems with transportation. The current findings demonstrate that there are no clear policies on the transfer of patients from one hospital to the other. This refers to different levels of operation like the district and regional/tertiary hospitals where the patients should be transferred. Lack of policies could result in chaos, as medical practitioners/doctors may do as they wish. This was contrary to the study by Nkurunziza et al. [23] on referral patterns and predictors of referral delays for injured patients in rural Rwanda, which showed that more than $50 \%$ of referrals were completed timely because there was strong referral system and patient support programme was in place [24].

Adherence to Basic Antenatal Care (BANC) audits was emphasized in the recommendations for the Saving Mothers Report. ANC aims at assessing for pregnancy problems and risks, diagnosis and treatment of problems arising during ANC, providing medication and information to improve pregnancy outcome and preparing the woman physically and psychologically for labour and parenthood. Participants in the study indicated that BANC audits were not done regularly and there was a limited follow-up on how BANC is conducted. ${ }^{25}$ Women were booking late, but the Guidelines for Maternity Care in South Africa states that pregnant women are expected to book for ANC as early as before 12 weeks of pregnancy. The guidelines [25] indicate that women are expected to visit the clinic 4 to 5 times during pregnancy. If the pregnancy is without risk factors, women are expected to visit the clinic at 20 weeks, 26 to 28 weeks, 32 to 34 weeks and 38 weeks. The woman should also visit the clinic at 41 weeks if still pregnant. For women who are at high risk, return visits are scheduled according to their specific challenges. The findings of the study showed that participants were not adhering to protocols; they indicated that pregnant women's health-seeking behaviours were problematic. In the study by Boltman-Binkowski [26] showed that most of the avoidable causes of deaths were patient-related, which included late booking and seeking medical intervention. Women, when booked late, they miss health education on obstetric emergencies to report to the midwives during their pregnancy. The midwife is expected to give pregnant women health education about the obstetric emergencies verbally or in a written form on danger signs and symptoms of pregnancy, which include: severe headaches, abdominal pain, and drainage of liquor through the vagina, vaginal bleeding and decreased foetal movements [27].

The Saving Mothers' recommendation number 4 on $5 \mathrm{Hs}$, namely; HIV, haemorrhage, hypertension, health worker training and health system strengthening, encourages the strengthening of the health facilities. The management of the facilities needs to support midwives in all challenges encountered when providing care. Findings revealed that participants expressed limited support and appreciation from the facility managers. This was in line with the results of the study by Banovcinova et al. [28] in Slovakia, which indicated that the midwives were burnout with low morale and because of conflicts with doctors, supervisors and colleagues. Low morale was cited to be due to work overload, which leads to emotional exhaustion. Authors [29]concurred that nurses had low moral due to their unfavourable conditions like work overload due to a shortage of manpower. The results also showed that visit by the manager to the ward would boost the morale of the staff. This is asserted by Hämmig [30] that the key role of supervisor support facilitates health and well-being at work. While Calvert [31] further pointed that support for midwives in New Zealand showed that there is a high possibility of leading and of feeling valued, which may increase their job satisfaction and reduce the turnover rate. These may, in turn, improve the care for childbearing women.

\section{LIMITATIONS}

The population of the study was limited to a small sample; only five participants per health facility in each district of Limpopo province. Findings cannot be generalised to all PHC facilities in Limpopo Province.

\section{CONCLUSION AND RECOMMENDATIONS}

Implementation of recommendations for Saving Mothers' report was hindered by the poor supply of resources, increased workload, problems associated with the referral system, nonadherence to protocols and limited appreciation by the management. Effective implementation of recommendations for Saving Mothers' report could contribute to the achievement of Sustainable Development Goal 3.1 and 3.2, by improving maternal health outcomes. As much as what was found seems to be similar to what was found previously, the findings and 
recommendations will be presented at the district's perinatal meeting to debate the applicability and implementability. It is recommended that referral policies and procedures be reinforced through workshops, monitoring and evaluation visits to ensure adherence by those involved in maternal health care. Further research is recommended on pregnant women's knowledge on their responsibilities during pregnancy, and women's behaviour as a contributory factor to maternal mortality.

\section{AUTHORS' CONTRIBUTIONS}

The project was conducted to fulfill the requirement of the doctoral degree qualification. The project leader was K.E.M a PhD student, M.S.M promoter; H.N.S and L.M.N copromoters, respectively. The student under supervision was involved in conceptualisation, data collection, analysis, and report writing and drafting of the manuscript, M.S.M, H.N.S and L.M.N augmented the literature search and refined the manuscript.

\section{ETHICS APPROVAL AND CONSENT TO PARTI- CIPATE}

This study has been approved from the Ethics Committee (Ref: SHS/16/PDC/01/2804) of University of Venda, South Africa.

\section{HUMAN AND ANIMAL RIGHTS}

Not applicable.

\section{CONSENT FOR PUBLICATION}

All patients participated on a voluntary basis and gave their informed consent.

\section{AVAILABILITY OF DATA AND MATERIAL}

The raw data used to support the findings of this study are included in the article and can also be obtained from the corresponding author upon request.

\section{FUNDING}

None.

\section{CONFLICT OF INTEREST}

The authors declare no conflict of interest, financial or otherwise.

\section{ACKNOWLEDGEMENTS}

The researchers would like to acknowledge the University of Venda, the Limpopo Provincial Department of Health and the Chief Executive Officers for permission to conduct this study. All registered midwives who participated in this study.

\section{REFERENCES}

[1] Moodley J, Pattinson R. Improvements in maternal mortality in South Africa. South African Medical Journal 2018; 108(3 Suppl 1): S4-8.

[2] Saving Mothers Fifth report on Confidential Enquiries into Maternal deaths in South Africa 2008-2010. Pretoria: Government Printers 2012.

[3] Saving Mothers 2014-2016: Seventh triennial report on confidential enquiries into maternal deaths in South Africa: Short report. Pretoria: Government Printers 2018.

[4] Millennium Development Goals. 2014. [Cited 25 June 2019]. Available from http://www.statssa.gov.za/wp-content/uploads/2014/02/MDGR Repor t_2013_Final.pdf

[5] Khan N, Pradhan MR. Identifying factors associated with maternal deaths in Jharkhand, India: a verbal autopsy study. J Health Popul Nutr 2013; 31(2): 262-71

[http://dx.doi.org/10.3329/jhpn.v31i2.16391] [PMID: 23930345]

[6] Mavalankar D, Singh A, Patel S, Desai A, Singh PV. Averting Maternal Death and Disability: Saving mothers and newborns through an innovative partnership with private sector obstetricians: Chiranjeevi Scheme of Gujarat, India. Int J Gynecol Obst 2009; (107): 271-6.

[7] Bar-Zeev S, Barclay L, Kruske S, Kildea S. Factors affecting the quality of antenatal care provided to remote dwelling Aboriginal women in northern Australia. Midwifery 2014; 30(3): 289-96.

[http://dx.doi.org/10.1016/j.midw.2013.04.009] [PMID: 23809580]

[8] World Health Organisation. World Health Statistics 2018: Monitoring health for Sustainable Development Goals (SDGs) 2018. [Cited 30 June 2019]. Available from https://www.who.int/gho/publications/ world_health_statistics/2018/en/

[9] Creswell JW, Clark VLP. Designing and Conducting Mixed Methods Research 2011. Thousand Oaks, United States: SAGE publishing Inc. 2011.

[10] Lincoln YS, Lynham SA, Guba EG. The handbook of Qualitative Research. Thousand Oaks, CA: Sage Publications 2011.

[11] Babbie ER, Mouton J. The practice of social research. Cape Town: Republic of South Africa, Oxford University Press Southern Africa 2007.

[12] Guidelines for Maternity Care in South Africa A manual for clinics, community health centres and district hospitals. Pretoria: Government Printers 2016.

[13] Gallaher JR, Mulima G, Kopp D, Shores CG, Charles AG. Consequences of centralised blood bank policies in sub-Saharan Africa. Lancet Glob Health 2017; 5(2): e131-2.

[http://dx.doi.org/10.1016/S2214-109X(16)30364-3] 28104174]

[14] du Plessis N. Obstetric haemorrhage and the use of blood and blood products. Obstet Gynaecol Forum 2013; 23: 15-21.

[15] Mazer-Amirshahi M, Goyal M, Umar SA, et al. U.S. drug shortages for medications used in adult critical care (2001-2016). J Crit Care 2017; 41: 283-8.

[http://dx.doi.org/10.1016/j.jcrc.2017.06.005] [PMID: 28622641]

[16] Fox ER, McLaughlin MM. ASHP guidelines on managing drug product shortages. Am J Health Syst Pharm 2018; 75(21): 1742-50. [http://dx.doi.org/10.2146/ajhp180441] [PMID: 30061155]

[17] Free State in Chains. Report back from People's Commission of Inquiry into Free State Health Care System 2015. Available at: https://tac.org.za/files/Free_State_In_Chains_-

Report_of_Peoples_Commission_of_Inquiry.pdf

[18] Phala MS. Assessment of medical equipment in the maternity unit at a district hospital of the Greater Tubatse Sub-district (unpublished dissertation). University of Witwatersrand. 2011.

[19] World Health Organisation. Working together for health 2006.https://www.who.int/whr/2006/en/

[20] Chopra M, Daviaud E, Pattinson R, Fonn S, Lawn JE. Saving the lives of South Africa's mothers, babies, and children: can the health system deliver? Lancet 2009; 374(9692): 835-46.

[http://dx.doi.org/10.1016/S0140-6736(09)61123-5] 19709729]

[21] Htwe O, Coates PD, Wint Z, Krasu M, Khin HY, Bidin H. Interhospital emergency obstetric referrals to the labour ward of RIPAS Hospital. Brunei Internal Medicine Journal 2011; 7: 22-33.

[22] Lee C, Aules Y, Sami S, Lar PK, Schlect J, Robinson C. Sexual and reproductive health needs and risks of very young adolescent refugees and migrants from Myanmar living in Thailand. Confl Health 2017; 11(Suppl. 1): 30-6.

[http://dx.doi.org/10.1186/s13031-017-0130-0] [PMID: 29163668]

[23] Nkurunziza T, Toma G, Odhiambo J, et al. Referral patterns and predictors of referral delays for patients with traumatic injuries in rural Rwanda. Surgery 2016; 160(6): 1636-44.

[http://dx.doi.org/10.1016/j.surg.2016.08.006] [PMID: 27743716]

[24] Murray SF, Pearson SC. Maternity referral systems in developing countries: current knowledge and future research needs. Soc Sci Med 2006; 62(9): 2205-15.

[http://dx.doi.org/10.1016/j.socscimed.2005.10.025] [PMID: 
16330139]

[25] Guidelines for Maternity Care in South Africa A manual for clinics, community health centres and district hospitals. Pretoria: Government Printers 2015.

[26] Boltman-Binkowski H. Assessment of quality of care in the management of postpartum haemorrhage: A review of selected maternal death cases. African J Physical, Health Education. Recreation Dance 2015; 1: 549-57.

[27] Hailu M, Gebremariam A, Alemseged F. Knowledge about obstetric danger signs among pregnant women in Aleta Wondo district, Sidamazone, Southern Ethopia. Ethiop J Health Sci 2010; 20(1): 25-32.

[PMID: 22434957]

[28] Banovcinova L, Baskova M. Sources of work-related stress and their effect on burnout in midwifery. Procedia Soc Behav Sci 2014; 132: 248-54.

[http://dx.doi.org/10.1016/j.sbspro.2014.04.306]

[29] Mudallal RH, Saleh MYN, Modallal HM, Abdel-Rahman RY. Quality of nursing care: The influence of work conditions, nurse characteristics and burnout. Int J Africa Nurs Sci 2017; 7: 24-30.

[http://dx.doi.org/10.1016/j.ijans.2017.06.002]

[30] Hämmig $\mathrm{O}$. Health and well-being at work: The key role of supervisor support. SSM Popul Health 2017; 3: 393-402.

[http://dx.doi.org/10.1016/j.ssmph.2017.04.002] [PMID: 29349232]

[31] Calvert I. Support for midwives - a model of professional supervision based on the recertification programme for midwives in New Zealand. Women Birth 2014; 27(2): 145-50.

[http://dx.doi.org/10.1016/j.wombi.2014.01.002] [PMID: 24486026]

\section{(C) 2020 Mothapo et al.}

This is an open access article distributed under the terms of the Creative Commons Attribution 4.0 International Public License (CC-BY 4.0), a copy of which is available at: https://creativecommons.org/licenses/by/4.0/legalcode. This license permits unrestricted use, distribution, and reproduction in any medium, provided the original author and source are credited. 